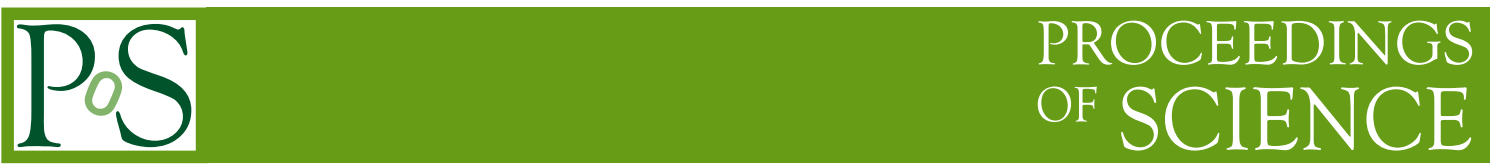

\title{
Latest developments in planar n-on-p sensors
}

\author{
Anthony AFFOLDER* \\ Department of Physics, Oliver Lodge Laboratory, University of Liverpool \\ Liverpool, L69 7ZE, United Kingdom \\ E-mail: affolderdhep.ph.liv.ac.uk
}

To further extend the ultimate physics reach of the experiments at the Large Hadron Collider (LHC), a series of accelerator and experimental upgrades are planned between 2014 and 2022. The final machine upgrade, called the High Luminosity-LHC (HL-LHC), is foreseen to increase the instantaneous luminosity by a factor ten with a targeted total integrated luminosity of $3000 \mathrm{fb}^{-1}$. To cope with the predicted high particle rates and intense radiation doses, $\mathrm{n}$-inp FZ planar technolgy has been developed with remarkable radiation tolerance. This technology might even be able to withstand the harsh environment at the innermost layers of the experimental upgrades for the HL-LHC.

This article summarizes the current understanding of the radiation tolerance of n-in-p FZ sensors. Plans and recent progress in prototyping silicon micro-strip and pixel devices at various foundries is shown. The different $R \& D$ efforts to reduce the inactive regions and to enhance the charge multiplication properties of such devices are also described.

The 20th Anniversary International Workshop on Vertex Detectors

June 19 - 24, 2011

Rust, Lake Neusiedl, Austria

* Speaker. 


\section{Introduction}

The planned upgrades to the Large Hadron Collider accelerator complex (High LuminosityLHC, HL-LHC) [U] provide many challenges to the silicon trackers of the experiments []. The factor of ten increase to the instantaneous luminosity requires much finer segmentation to retain efficient track finding. The targeted integrated luminosity of $3000 \mathrm{fb}^{-1}$ over the ten year lifespan of the HL-LHC increases the needed radiation tolerance for the silicon sensors. The estimated end-of-lifetime fluence for the innermost layer of an experimental upgrade is approximately $2 \times 10^{16} \mathrm{n}_{\mathrm{eq}} \mathrm{cm}^{-2}$ [B]], given in $1 \mathrm{MeV}$ neutron equivalent particles per square centimeter $\left(\mathrm{n}_{\mathrm{eq}} \mathrm{cm}^{-2}\right)$. The need for much more radiation tolerant silicon sensors was foreseen, with the RD48 collaboration [䧃] and its successor (RD50 [5] ) developing n-implant readout planar silicon sensors for the LHC experiments [ $[$,, , , 8$]$ and the HL-LHC experimental upgrades, respectively.

The current inner layers of the LHC experiments utilize segmented $n$-implant readout in n-bulk float zone technology (n-in-n FZ). Recent studies have changed focus to p-bulk FZ silicon (n-in-p FZ) as it has a number of advantages relative to n-in-n FZ technology: it always depletes from the segmented side; it is a single-sided production process with more foundries and more available capacity world-wide; it can be $\sim 50 \%$ cheaper than n-in-n FZ devices; and it is easier to handle and test due to the lack of a patterned back-side implant.

This paper focuses on the development of planar n-in-p FZ sensor technology into experimental systems for the HL-LHC experimental upgrades. First, a short summary of the current understanding of the outstanding radiation tolerance of this technology will be given. Current and future prototype submissions of n-in-p FZ planar silicon devices to different foundries is presented. Finally, the R\&D efforts to reduce the inactive regions and to enhance the charge multiplication properties of such devices are described.

\section{Current Understanding of Performance After Irradiation}

$\mathrm{N}$-implant readout (n-in-n, n-in-p) was initially believed to be more radiation tolerant than the more standard p-in-n geometry due to the following: after irradiation, the dominant junction in the double junction field $[9,[0]$ is located underneath the n-implant, which is also the location of the maxima of the weighting field; electron mobility is larger than hole mobility, which results in shorter collection times and bigger signals in regimes where charge trapping is significant; and the charge trapping constants for electrons are smaller than for holes [ㄴ], ㅁ2, [3], ㄴ4]. At lower fluences $\left(<1 \times 10^{15} \mathrm{n}_{\mathrm{eq}} \mathrm{cm}^{-15}\right)$, experimental measurements match simulations based on these assumptions and on the measured full depletion voltages [ㄷ]].

At higher fluences, experimental measurements do not match the expectations from simulation. The collected charge of n-in-p FZ devices was shown to be much higher than expected, with a collected charge of over $8 \mathrm{ke}^{-}$measured after a fluence of $1 \times 10^{16} \mathrm{n} \mathrm{cm}^{-2}$ at $1000 \mathrm{~V}$ [ए6]. Irradiated silicon devices using different bulk types (n-type and p-type doping) and growth techniques (floating zone and magnetic Czochralski) were studied. The collected charge for the different devices became very similar above $2 \times 10^{15} \mathrm{n}_{\mathrm{eq}} \mathrm{cm}^{-2}$ after reactor neutron [ए]] and $26 \mathrm{MeV} / \mathrm{c}$ proton irradiations [1[8], as shown in fig. W. More than full charge collection efficiency was observed at large bias voltages in $300 \mu \mathrm{m}$ thick n-in-p FZ devices irradiated up to $3 \times 10^{15} \mathrm{n}_{\mathrm{eq}} / \mathrm{cm}^{-2}$ [एW] . 
Charge multiplication effects were then conclusively demonstrated in $100 \mu \mathrm{m}$ and $150 \mu \mathrm{m}$ thick high resistivity epitaxial diodes [20] and in $140 \mu \mathrm{m}$ thick n-in-p FZ micro-strips [2]]. Fig. W shows the collected charge as a function of voltage for $140 \mu \mathrm{m}$ and $300 \mu \mathrm{m}$ thick n-in-p FZ devices after irradiation with $26 \mathrm{MeV} / \mathrm{c}$ protons to a fluences of $5 \times 10^{15} \mathrm{n}_{\mathrm{eq}} \mathrm{cm}^{-2}$; the collected charge is more than 2 times the expectations from minimum ionizing particle for the thinner device. These results imply that the electric field is much larger than previously expected over the entire volume of the devices (denoted active base) and that charge multiplication is enhancing the collected charge at these high fluences and large bias voltages.
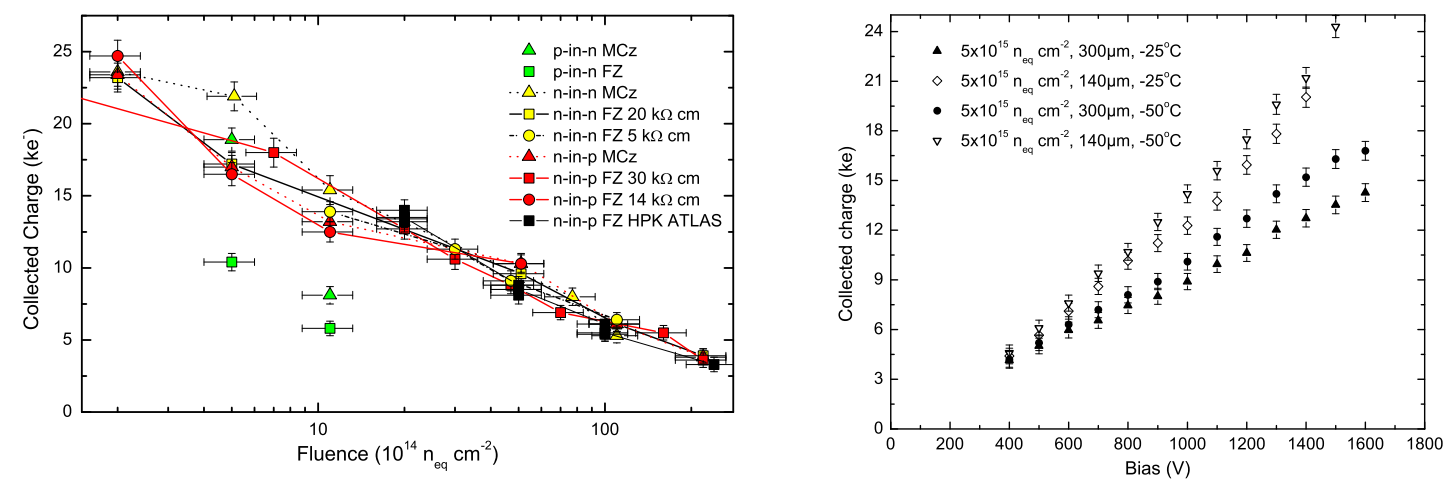

Figure 1: Left: The collected charge as a function of fluence after $26 \mathrm{MeV} / \mathrm{c}$ protons at a bias voltage of $900 \mathrm{~V}$ for different $300 \mu \mathrm{m}$ thick substrate geometries [ष्ष]. Right: The collect charge as a function of bias voltage after irradiation with $26 \mathrm{MeV} / \mathrm{c}$ protons to a fluence of $5 \times 10^{15} \mathrm{n}_{\mathrm{eq}} \mathrm{cm}^{-2}$ for $140 \mu \mathrm{m}$ and $300 \mu \mathrm{m}$ thick n-in-p FZ micro-strip devices [R]].

Edge-TCT measurements [22] show experimentally that both the active base and charge multiplication effects are presented; the effects determine the charge collection performance of planar silicon devices after $2 \times 10^{15} \mathrm{n}_{\mathrm{eq}} \mathrm{cm}^{-2}$. In edge-TCT, a pulsed, narrow laser beam deposits charge at a known depth within a sensor. Fast electronics resolve the shape of the induced signal with sub-100 ps resolution. The left side of fig. $\square$ shows the drift velocity of charge carriers as a function of depth and bias voltage for a device irradiated to $5 \times 10^{15} \mathrm{n} \mathrm{cm}^{-2}$. The large drift velocities at all depths and bias voltages measured imply much larger fields throughout the device than would be expected from full depletion measurements. The right side of fig. $\square$ shows the induced current as a function of time and laser depth for the same device at a bias voltage of $750 \mathrm{~V}$. The second peak in time is induced from holes produced by impact ionization of electrons near the $n^{+}$implant. Additional edge-TCT measurements are needed to cover the full range of fluences, device thicknesses and implant geometries of interest for the HL-LHC in order to better determine the electric field configurations for simulation models.

\section{Production Projects}

This technology is rapidly becoming utilized with a large number of prototype n-in-p FZ devices already submitted or produced for pixel and strip upgrades of ATLAS, CMS, and LHCb. Table $\square$ gives an overview of these submissions; 8 different foundries are currently involved in planar n-in-p FZ productions, most for the HL-LHC upgrades. As shown in [16, [20, 21], 22], thinner 

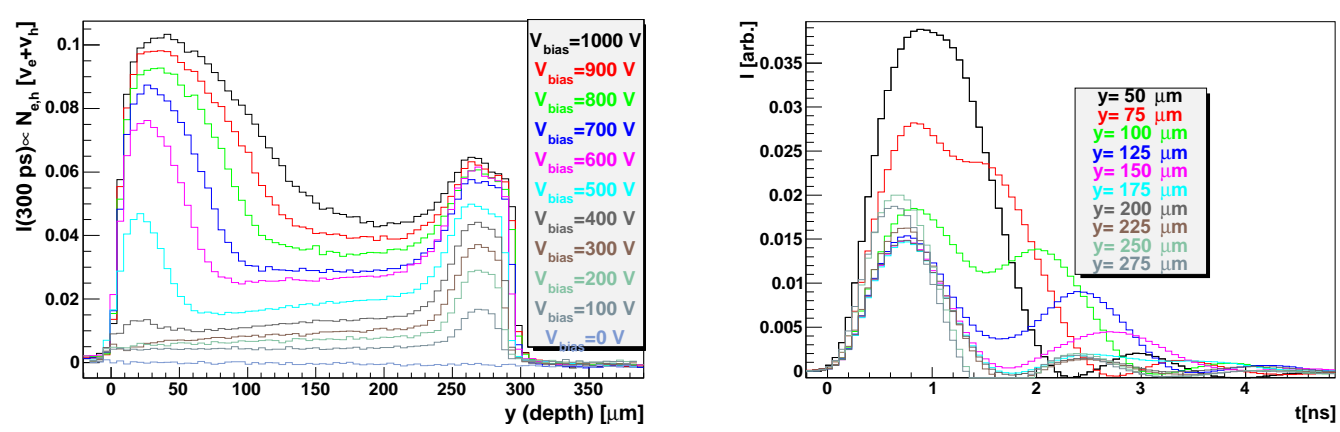

Figure 2: Left: The drift velocity of the charge carriers extracted from Edge-TCT measurements as a function of depth and applied bias voltage for a device irradiated to $5 \times 10^{15} \mathrm{n} \mathrm{cm}^{-2}$. Right: The induced current waveforms using Edge-TCT at different injection depth for a n-in-p FZ device with 750 bias irradiated to $5 \times 10^{15} \mathrm{n} \mathrm{cm}^{-2}$ [2]].

devices have larger collected charge at the high, end-of-lifetime fluences expected at the HL-LHC; therefore, many of these productions are studying multiple wafer thicknesses in order to optimize performance. Due to space-limitations, only a flavor of these production can be shown in next few sections of this proceeding.

\begin{tabular}{|c|c|c|c|}
\hline Foundry & $\begin{array}{c}\text { Wafer Sizes } \\
(\text { Inch })\end{array}$ & $\begin{array}{c}\text { Thicknesses } \\
(\mu \mathrm{m})\end{array}$ & Productions \\
\hline CiS & 4 & $150 / 200 / 285$ & ATLAS pixels, CMS pixels, RD50 \\
IMB-CNM & 4 & $150 / 300$ & ATLAS endcap strips, LHCb pixels, RD50 \\
e2v & 6 & 300 & ATLAS barrel strips \\
FBK & 4 & 200 & ATLAS pixels \\
Hamamatsu & 6 & $150 / 200 / 320$ & ATLAS pixels/strips, CMS pixels/strips, LHCb strips \\
Micron & $4 / 6$ & $150 / 300 / 500$ & ATLAS pixels, LHCb strips/pixels, RD50 \\
MPP-HLL & 6 & $75 / 150$ & ATLAS strips/pixels \\
VTT & 6 & $100 / 200 / 300 / 500$ & ATLAS pixels, LHCb pixels \\
\hline
\end{tabular}

Table 1: Active n-in-p foundries with high-energy physics projects

\subsection{LHCb Vertex Locator Sensors (Micron)}

Currently, there is only one pair of n-in-p FZ sensors utilized at the LHC. The LHCb Vertex Locator (VELO) [8] predominately consists of modules with pairs of n-in-n FZ sensors (one R measuring, one phi measuring), produced by Micron Semiconductor, Ltd (United Kingdom). The module farthest from the rest of the experiment uses two n-in-p FZ sensors. As of now, the performance of the $n-i n-n$ and n-in-p FZ devices is identical [[24], apart from the full depletion voltage as the $n$-in-p devices do not invert.

As the active elements of the VELO sensors are only $8 \mathrm{~mm}$ away from stable beams, an entire set of replacement modules is being produced using n-in-p FZ sensors. The p-type devices cost $30 \%$ less than the original n-type devices, even with the use of routing double-metal on the segmented n-implant side. The production has progressed well with half of the modules already delivered to CERN. The remainder of the modules are expected to be deliverd by autumn. 


\subsection{ATLAS Upgrade Strips Sensors (Hamamatsu)}

For the strip regions of the planned all-silicon HL-LHC tracker for ATLAS [25], planar n-in-p FZ devices have already been chosen to be the baseline technology. In collaboration of Hamamatsu Photonics K. K. (Japan), ATLAS has developed $9.75 \times 9.75 \mathrm{~cm}^{2}$ sensors suitable for the short strip regions of the barrel, which use the maximal area of 6 inch silicon wafers. The devices, denoted ATLAS07 [R6], are $310 \mu \mathrm{m}$ thick, and are divided into four $2.39 \mathrm{~cm}$ long segments ( 2 axial and 2 stereo with a $40 \mathrm{mrad}$ angle) with the 1280 strips each. 24 miniature $\left(1 \times 1 \mathrm{~cm}^{2}\right)$ micro-strip devices surrounding the main sensor have been utilized to study of the evolution of the silicon properties with irradiation. The full-size ATLAS07 sensors have been throughly evaluated within the collaboration [27] with all bulk and surface properties within the technical specifications prior to irradiation.

An extensive program of ATLAS07 miniature sensors irradiations has been undertaken with reactor neutrons from the TRIGA reactor in Ljubljana, Slovenia [28], charged pions from the PiE1 beamline of the proton accelerator at the Paul Scherrer Institut in Villigen, Switzerland [2Q] and protons from the Compact Cyclotron at Karlsruhe, Germany [B] ] and from the the Cyclotron Radio Isotope Centre (CYRIC) at Tohoku University, Japan [B]]. The evolution of the sensor properties as a function of particle fluence due to bulk (depletion voltage, leakage current, and collected charge) [B2] and surface damage effects (interstrip resistance, interstrip capacitance and punch-through protection voltage) [33], B3] has been measured.

Fig. [3] shows new results after pion irradiations and summary of all charge collection measurements made with ATLAS07 miniature sensors at $900 \mathrm{~V}$ as a function of fluence. For devices that were temperature annealed, the collected charge is corrected back to a pre-annealed state using factors derived from [35]. The collected charge for all irradiation sources after the NIEL corrections are consistent within the uncertainties on the fluence and collected charge. The measured values are also consistent with previous measurements of irradiated Micron devices to the same fluences.
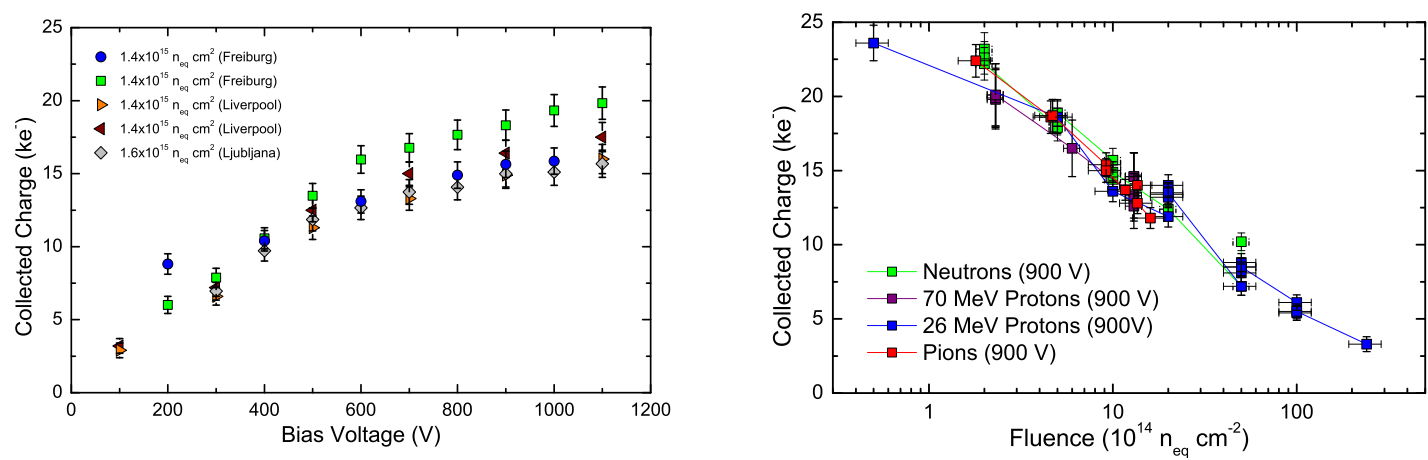

Figure 3: Left: Collected charge of ATLAS07 micro-strip sensors produced by Hamamatsu as a function of bias voltage after $280 \mathrm{MeV}$ pion irradiation and annealing the devices for 80 minutes at $60 \mathrm{C}^{\circ}$. Right: Summary of collected charge of the ATLAS07 devices as a function of $1 \mathrm{MeV} \mathrm{n}$ eq fluences for the different irradiation sources studied at a bias voltage of $900 \mathrm{~V}$. The measurements are performed without temperature annealing or corrected back to an pre-annealing state.

\subsection{CMS Upgrade Joint Strip-Pixel Sensors (Hamamatsu)}

The CMS collaboration is undertaking an extensive program [B] of sensor studies of devices 
produced by Hamamatsu. As shown in fig. 田, the 6 inch mask includes diodes, miniature strip and pixel structures with different geometries, and double-metal and Lorentz angle test structure. Float zone, magnetic Czochralski and epitaxially grown bulk materials in both n-in-p and p-in$\mathrm{n}$ geometries will be studied in multiple thicknesses. For the $\mathrm{n}$-in-p devices, the project is also investigating both p-spray and p-stop strip isolation techniques. The devices will be characterized prior to and after mixed particle type irradiations, with both surface and bulk properties evaluated. As the devices will be produced by one vendor and will be tested using an uniform protocol, it is hoped that most systematic uncertainties in the comparison of the different materials and thicknesses will be reduced to a minimum. In total, 158 wafers will be produced at a cost of 200 $\mathrm{kCHF}$ with $\sim 30$ structures per wafer to be evaluated.

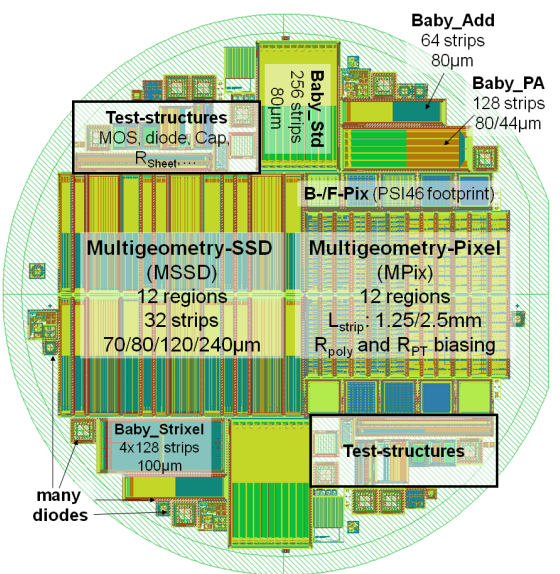

\begin{tabular}{|c|c|c|c|c|}
\hline bulk-type & $\begin{array}{c}\text { thickness } \\
(\mu \mathrm{m})\end{array}$ & $\begin{array}{c}\text { n-type } \\
\text { (p-stop) }\end{array}$ & $\begin{array}{c}\text { p-type } \\
(\mathrm{p} \text {-spray) }\end{array}$ & p-type \\
\hline FZ & 320 & 6 & 6 & 6 \\
FZ deep diff. & 200 & 6 & 6 & 6 \\
FZ deep diff. & 120 & 6 & 6 & 6 \\
MCz & 200 & 6 & 6 & 6 \\
Epi & 100 & 2 & 6 & 6 \\
Epi & 70 & 4 & & \\
Epi & 50 & 6 & 6 & 6 \\
FZ deep diff. & & & & \\
\& double metal & 200 & 6 & 6 & 6 \\
FZ & 200 & 6 & 6 & 4 \\
FZ on carrier & 120 & 6 & 6 & 4 \\
\hline
\end{tabular}

Figure 4: Left: The mask layout of the CMS joint strip-pixel densor submission with Hamamatsu Photonics, Ltd. Right: Table of number of wafers, substrate bulk type and substrate thicknesses included in the CMS joint strip-pixel sensor production.

\subsection{ATLAS Upgrade Strips Sensors (e2v)}

In order to qualify another large-volume silicon vendor for the strip region of the HL-LHC upgrade, ATLAS has been producing $300 \mu$ m planar n-in-p FZ sensors on 6 inch wafers with e2v Technologies, plc (United Kingdom). The first submission consisted entirely of miniature sensors $\left(1 \times 1 \mathrm{~cm}^{2}\right)$, which were used to determine the optimal strip isolation and passivation techniques. The preliminary results are encouraging; a set of parameters have been found which yields acceptable performance prior to irradiation with a collected charge after $24 \mathrm{GeV}$ proton irradiation [B]] consistent with previous measurements, see fig. [1. Axial-only, full-size $\left(9.75 \times 9.75 \mathrm{~cm}^{2}\right)$ sensors in the ATLAS short strip geometry are currently under production which will be compatible with both the 128 channel, $0.25 \mu \mathrm{m}$ CMOS readout ASIC (ABCN25) and the final 256 channel, 130 nm CMOS readout ASIC (ABCN13).

\subsection{RD50-CMS-ATLAS Upgrade Pixels Sensors (CiS)}

One concern with using n-in-p FZ devices with hybrid-pixels is the large potential difference between the sensor's edges and the readout ASIC, which could cause electrical discharges that damage the pixel package. In order to address this concern, a joint ATLAS-CMS pixel production 

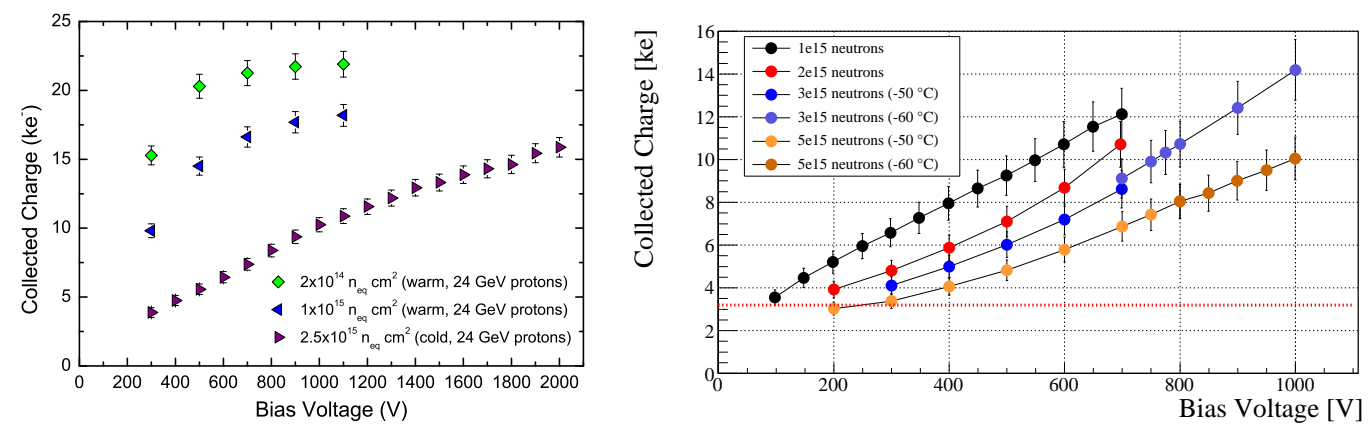

Figure 5: Left: Collected charge as a function of bias voltage for $\mathrm{e} 2 \mathrm{v}$ micro-strip devices irradiated with $24 \mathrm{GeV} / \mathrm{c}$ protons. Right: Charge collection as a function of bias voltage for $285 \mu \mathrm{m}$ thick, ATLAS FE-I3 pixel devices produced by CiS [B]]. The sensor surface is covered in $3 \mu \mathrm{m}$ of BCB at IZM Berlin in order to improve the strip edge isolation.

has been undertaken within the RD50 collaboration with CiS Institut für Mikrosensorik and IZMBerlin [B8] $285 \mu \mathrm{m}$ thick sensors compatible with the ATLAS FE-I3 and CMS ROC pixel ASICs are produced by $\mathrm{CiS}$, and then the sensor surface is covered with $3 \mu \mathrm{m}$ of Benzocyclobutene (BCB) by IZM Berlin. The BCB layer increases the isolation between the sensor's edges and the front end ASICs. A number of FE-I3 pixel packages have been bump bonded by IZM-Berlin and then irradiated with reactor neutrons [ [R8]. The BCB post-processing has been successful, with $1000 \mathrm{~V}$ bias applied for several hours before and after irradiation with no sign of breakdown. As shown in fig. [1, the collected charge after irradiation using the FE-I3 pixel ASIC is consistent with previous n-in-p FZ micro-strip measurements.

$\mathrm{CiS}$ is also producing another pixel sensor wafer for ATLAS. Both 1- and 2-chip devices compatible with the new FE-I4 readout ASIC are included. The FE-I4 is planned to be used in the Insertable B-Layer (IBL) upgrade in 2014 and the outer pixel upgrade in 2021. The sensors will be produced in three thicknesses $(150,200$, and $300 \mu \mathrm{m})$ without a handle wafer. The devices will be used to study the effects of thickness on collected charge, and on the sensor and bump bonding yields.

\subsection{ATLAS Upgrade Pixel Sensors (Micron)}

There are a number of difficulties testing irradiated hybrid pixel packages: bump bonding of a small number of prototypes is expensive and it is difficult to disentangle the irradiation effects of the sensor and readout ASIC. In order to answer both of these issues, a pixel sensor wafer has been designed by ATLAS and produced by Micron. The 6 inch wafers contain standard pixel devices which match to ATLAS FE-I3, CMS PSI-46 and Medipix readout ASICs as well as a set of bondable pixels. For the bondable devices, 8 interleaved pixel sets are connected together using a double-metal layer which ends in a wire-bondable pad. These devices can be irradiated separately from any electronics and then wire bonded to LHC-speed, analogue readout ASICs with minimal heating. The first tests [39] of these devices after neutron irradiations were successful with the collected charge consistent with previous measurements of micro-strip devices.

A second set of 6 inch pixel wafers have also been produced by Micron for ATLAS in 140 and $300 \mu \mathrm{m}$ thicknesses without a handle wafer. This wafer consists of a large number of 1- and 
2-FE-I4 compatible sensors. The devices are now at IZM for under-bump metalization and bump bonding to FE-I4s.

\subsection{ATLAS Upgrade Thin Strip-Pixel Sensors (MPP-HLL)}

Max-Planck-Institut für Physik and MPI Halbleiterlabor have undertaken a joint project (MPPHLL) to make thin (75 and $150150 \mu \mathrm{m}$ ) micro-strip and pixel sensors compatible with the ATLAS FE-I3 pixel chip using a handle wafer. The handle wafer can then be selectively etched to act as a thin support frame and to increase stiffness with minimal material. These produced devices had an excellent $99 \%$ yield, low bias currents $\left(\sim 10 \mathrm{nA} / \mathrm{cm}^{2}\right)$ and good breakdown behaviour. Three micro-strip devices of each thickness were irradiated with $26 \mathrm{MeV} / \mathrm{c}$ protons to fluences up to $1 \times 10^{16} \mathrm{n}_{\mathrm{eq}} \mathrm{cm}^{-2}$. As shown in fig. 6 , the devices performed extremely well with full charge collection efficiency still possible for the thinner devices for all the fluences studied. [40]].

\subsection{ATLAS Upgrade Thin Pixel Sensors (Hamamatsu)}

Finally, ATLAS is also producing thin n-in-p FZ pixel sensors [4]] with Hamamatsu. The 6 inch wafers contain 1- and 4-chip FE-I3 compatible devices and 1- and 2-chip FE-I4 compatible devices. The front of the wafers are patterned on $320 \mu \mathrm{m}$ thick wafers; after finishing the front, the wafers are thinned to $150 \mu \mathrm{m}$ and then the backside is completed. As shown in fig. 6 , the I-V performance of these devices before irradiation is remarkable with the first signs of breakdown above $900 \mathrm{~V}$. A number of FE-I4 devices are at IZM ready for bump bonding.
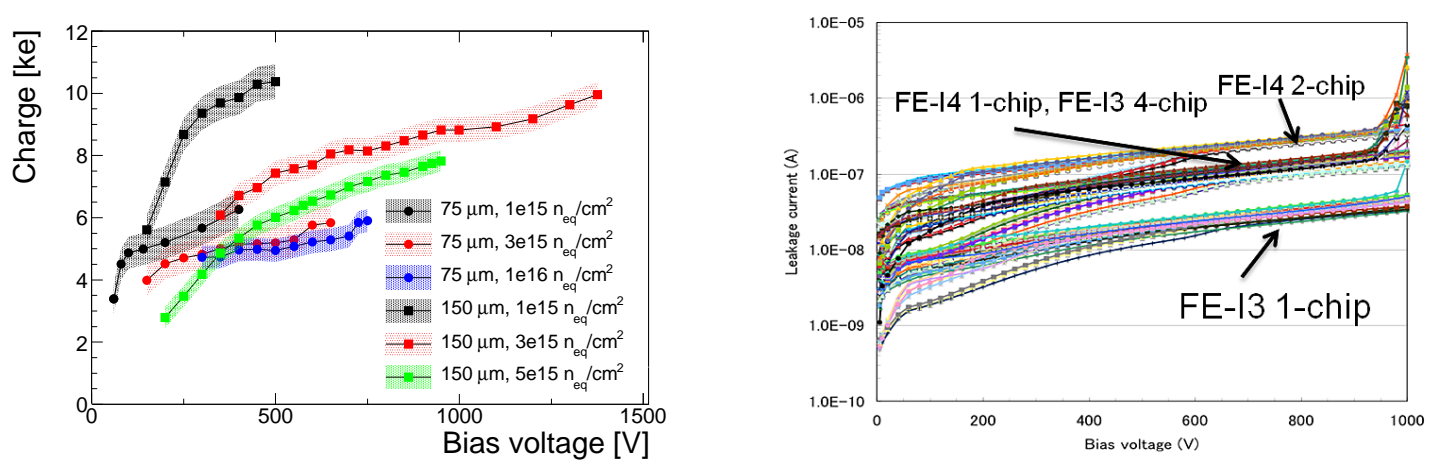

Figure 6: Left: Charge collection of MPP-HLL strip devices as a function of voltage after irradiation with $26 \mathrm{MeV} / \mathrm{c}$ protons [40]. Right: Leakage current vs. bias voltage for $140 \mu \mathrm{m}$ thick pixel devices produced by Hamamatsu. The differences in the leakage current scaled with the areas of the different device types.

\section{Research and Development Studies}

In addition to wafer productions intended for HL-LHC experimental upgrade prototyping, an active program of device $R \& D$ is in progress on two main fronts: edge reduction and charge multiplication enhancement. Tiling of pixel detectors edge-to-edge is attractive in order to reduce material in overlapping devices; tiling puts a premium on reducing the inactive sensor edges as particles crossing these areas would not be detected in that particular layer of the system. Charge multiplication is now understood to be an important element to the radiation tolerance of planar 
silicon sensors; as such, efforts have begun to try to understand how device geometry influence charge multiplication properties in an attempt to make more radiation tolerant devices.

\subsection{Edge Reduction}

As part of the production of ATLAS pixel devices with Hamamatsu, special 'slim-edge' diodes were fabricated. These diodes have 3 edges with the standard Hamamatsu diode layout and the last edge being narrowed. As shown in fig. $\square$, a linear relationship between the total edge width and the square root of the breakdown onset voltage is measured [ब⿴囗十]]. Because of the square root dependence of the onset voltage, it is believe the onset of breakdown is caused by lateral depletion along the surface of the sensor reaching the device's cut edge. After irradiation, the onset voltage increases. With the results of this study, the minimal edge size needed for a given use-case can be estimated.
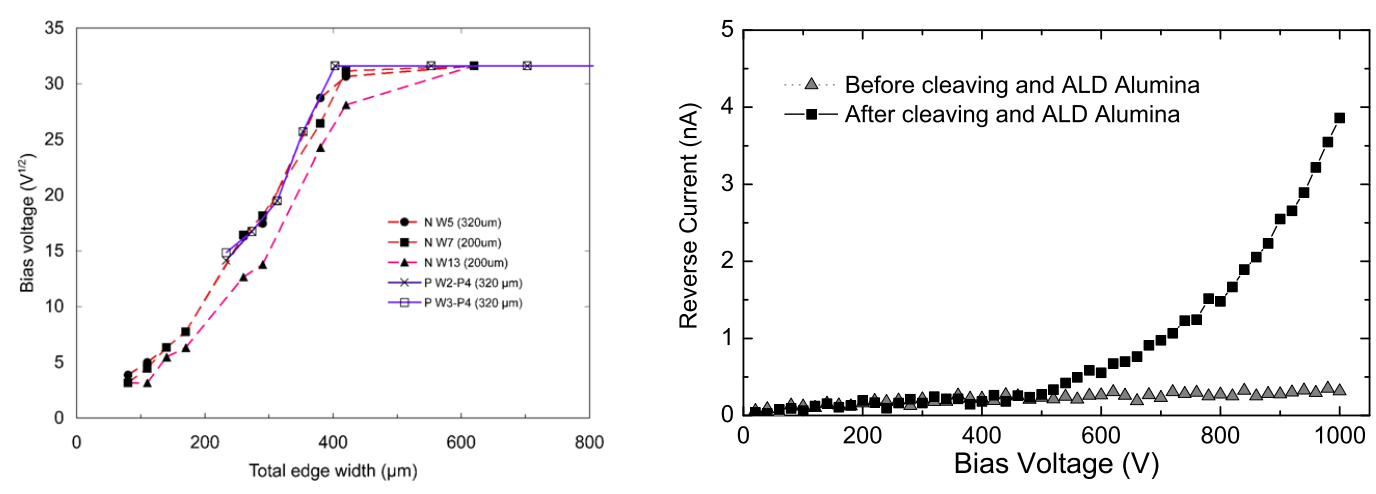

Figure 7: Left: The square root of the bias voltage for the start of breakdown as a function of the total edge width for Hamamatsu test devices. Right: the leakage current as a function of bias voltage for an ATLAS07 diode with cleaved edges $54 \mu \mathrm{m}$ from the guard ring and ALD alumina edge passivation,

In high-efficiency solar cells, passivation with alumina using an atomic layer deposition (ALD) process provides field-induced isolation of the device edges, due to negative trapped charges in the alumina [42]. The University of California at Santa Cruz has been investigating the use of ALD alumina for the sidewall passivation after laser scribing and cleaving of n-in-p FZ planar sensors to produce slim edges. Results so far have been really promising; a pad diode from the ATLAS07 series has been cleaved $54 \mu \mathrm{m}$ within the single guard ring and passivated with ALD alumina. After an high-temperature annealing step, the device has low leakage currents up to $1000 \mathrm{~V}$ [43], as shown in fig. $\square$. Based on these results, a new RD50 common project has been funded to extend this study to pixel and strip sensors with both n-type and p-type bulk, including basic measurements of the charge density profile on the edges, of surface recombination and lifetime measurements at the cleaved edges, and of SEM images of the cleaving and ALD alumina.

The final approach for reducing the inactive volume of devices is active edges. After backside implantation, sensors are oxide bonded to a support wafer. The edges of each device is defined by deep reactive-ion etched trenches with $p^{+}$doping. The trenches are then filled with polysilicon. As shown in fig. $\mathbb{8}$, this process leads to an active edge with the same doping as the backplane. Two sites are pursuing this option. FBK [44] in conjunction with LPNHE is designing FEI3 and FEI4 
compatible devices with no or three guard rings. VTT [45] is currently collecting partners for a joint active edge detector project with available thicknesses of 100 and $200 \mu \mathrm{m}$.
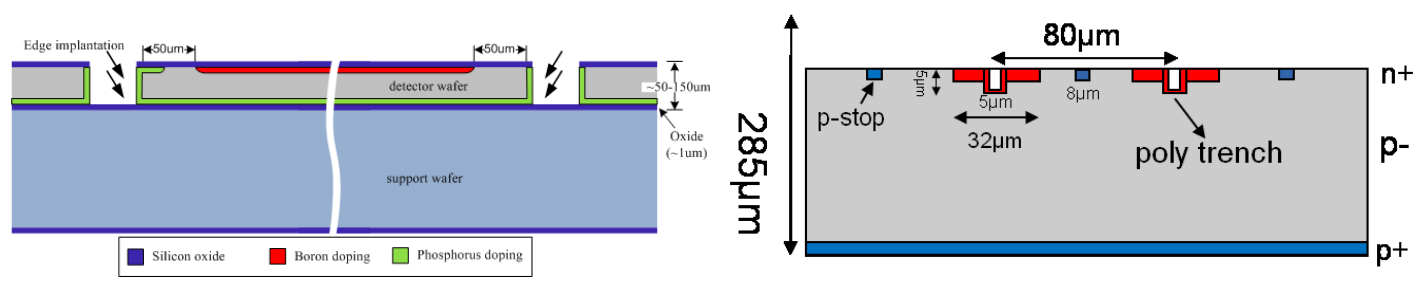

Figure 8: Left: Diagram of the active edge process at VTT. Right: Cross-sectional drawing of trenched detector for multiplication enhancement.

\subsection{Charge Multiplication Enhancement}

As most of the charge multiplication in planar n-in-p FZ sensors occurs in a narrow region around the segmented $n^{+}$implants, the shape of the implants may be able to be profiled to enhance or reduce this effect. In order to study the effects of implant geometry, the RD50 collaboration is producing $300 \mu \mathrm{m}$ thick, 6 inch n-in-p FZ wafers with Micron consisting of 60 miniature $\left(1 \times 1 \mathrm{~cm}^{2}\right)$ strip sensors. The devices have 3 different pitches $(40,80$, and $100 \mu \mathrm{m})$; each pitch has 3 different implant widths. In addition, all the devices have variants with floating and biased intermediate strips. The wafers have been received and a large program of irradiation and measurement is underway.

Within RD50, a second production exploring enhancement of charge multiplication is underway at IMB-CNM. Two styles of miniature micro-strip devices are understudy. In the first style, a narrow trench is made along the center of the $n^{+}$implant and then filled with polysilicon doped with phosphorus, as shown in fig. 8 . This new $n^{+}$implant is expected to modify the electric field, increasing the rate of multiplication after irradiation. Trenches of 5,10 , and $50 \mu \mathrm{m}$ will be evaluated. In the second style, a p-implant is deeply diffused under the segmented $n^{+}$electrodes. The $\mathrm{n} / \mathrm{p}$ junction created along the center of the strips by the deeply diffused $n^{+}$implant creates a high field region, which could also enhance charge multiplication effects after irradiation. Simulations of these devices can be found at [46]. A number of these devices have been produced and irradiated. A study of the charge collection performance as a function of irradiation for the two device types is currently underway.

\section{Conclusion}

Enormous progress has been made evaluating n-in-p FZ technology. It has been shown to be radiation tolerant enough for even the innermost layers of HL-LHC experimental upgrades. Numerous productions of n-in-p FZ strip and pixel silicon devices with 8 different foundries are well underway, with n-in-p FZ technology already chosen as the baseline for the silicon strip regions of the ATLAS HL-LHC upgrades. Vibrant R\&D programs are underway to reduce the inactive regions of n-in-p FZ planar devices and to enhance the radiation tolerance through control charge multiplication. These avenues of research should result in the needed devices for the extreme radiation environments of HL-LHC experiments. 


\section{Acknowledgments}

Much of this work has been performed within the framework of the RD50 collaboration. In particular, the following RD50 common fund projects have supported the development of n-in-p FZ devices: 2005-03, 2005-04, 2007-03, 2008-01, 2009-01, 2009-03, 2010-01, 2010-03, 2011-03 and 2011-04.

The author would like to thank the team which arranged and performed the $280 \mathrm{MeV}$ pion irradiations at the proton accelerator of the Paul Scherrer Institut, Villigen, Switzerland. Beamline support was provided by Dr. D. Renker, Dr. K. Deiters; logistics and irradiation support provided by M. Glaser, C. Betancourt and M. Gerling. The author would also like to thank the team at the University of Karlsruhe, especially Dr. A. Dierlamm and Prof. W. de Boer, for providing the 26 $\mathrm{MeV}$ proton irradiations of the devices at the Compact Cyclotron in Karlsruhe. The authors would like to thank the team at the University of Ljubljana and especially Prof. V. Cindro who performed the neutron irradiations, sometimes at short notice. The author would also like to thank M. Glaser and F. Ravotti for their assistance for the $24 \mathrm{GeV}$ proton irradiation of the devices at the CERNIRRAD1 facility. The team of CYRIC, Tohoku University are also acknowledged for conducting excellent $70 \mathrm{MeV}$ proton irradiations.

\section{References}

[1] E. Tsesmelis, Future colliders at CERN, Fortschr. Phys. 58 (2010) 628.

[2] F. Gianotti et. al., Physics Potential and Experimental Challenges of the LHC Luminosity Upgrade, Eur. Phys. J. C39 (2005) 293.

[3] I. Dawson, Radiation Predictions at the SLHC and Irradiation Facilities, presented at the ATLAS Tracker Upgrade Workshop, Liverpool, 6-8 Dec 2006, http://www.liv.ac.uk/physics/AHLUTW/

[4] G. Lindstroem, et. al., Radiation hard silicon detectors developments by the RD48 (ROSE) collaboration, Nucl. Instr. Meth. A 466 (2001) 308.

[5] J. P. Balbuena, et. al., "RD50 Status Report 2008: Radiation Hard Semiconductor Devices for Very High Luminosity Colliders," CERN RD50 Collaboration, Geneva, Switzerland, CERN-LHCC-2010-012, Jan. 2009.

[6] G. Aad, et. al., ATLAS pixel detector electronics and sensors, JINST 3 (2008) P07007.

[7] Y. Allkofer, et. al., Design and performance of the silicon sensors for the CMS barrel pixel detector, Nucl. Instr. Meth. A 584 (2008) 25.

[8] A. Alves, et. al., The LHCb Detector at the LHC, JINST 3 (2008) S08005.

[9] L.J. Beattie, et. al., The Electric Field in Irradiated Silicon Detectors, Nucl. Instr. Meth. A, 418/2 (1998) 314.

[10] G. Casse, M. Glaser, \& E. Grigoriev, Study of evolution of active volume in irradiated silicon detectors, Nucl. Instr. Meth. A 426 (1999) 140.

[11] G. Kramberger, V. Cindro, I. Mandic, M. Mikuz, and M. Zavrtanik, Effective trapping times of electrons and holes in different silicon materials irradiated with neutrons, protons and pions, Nucl. Instr. Meth. A 481 (2002) 297. 
[12] G. Kramberger, et. al., Annealing Studies of Effective Trapping Times, Nucl. Instr. Meth. A 418/2 (2007) 608 .

[13] G. Kramberger, et. al., Influence of trapping on silicon microstrip detector design and performance, IEEE Trans. Nucl. Sci. 49 (2002) 1717.

[14] J. Weber \& R. Klingenberg Free charge carriers trapping properties in neutron-irradiated DOFZ silicon pad detectors, IEEE Trans. Nucl. Sci. 54 (2007) 2701.

[15] G. Kramberger, et. al., Comparison of pad detectors produce on different silicon materials after irradiation with neutrons, protons and pions, Nucl. Instr. Meth. A 612 (2010) 288.

[16] G. Casse, A. Affolder, \& P. Allport, Charge Collection Efficiency Measurements for Segmented Silicon Detectors Irradiation to $1 \times 10^{16} \mathrm{n} \mathrm{cm}^{-2}$, IEEE Trans. Nucl. Sci. 55 (2008) 1695.

[17] A. Affolder, et. al., Charge collection efficiencies of planar silicon detectors after reactor neutron and proton doses up to $1.6 \times 10^{16} n_{e q} \mathrm{~cm}^{-2}$, Nucl. Instr. Meth. A 612 (2010) 470 .

[18] A. Affolder, P. Allport \& G. Casse, Collected charge of planar silicon detectors after pion and proton irradiation up to $2.2 \times 10^{16} n_{e q} \mathrm{~cm}^{-2}$, Nucl. Instr. Meth. A 623 (2010) 177.

[19] I. Mandic, V. Cindro, A. Gorisek, G. Kramberger, M. Mikuz, M. Zavrtanik, Observation of full charge collection efficiency in heavily irradiated $n+p$ strip detectors irradiated up to $3 \times 10^{15} n_{\text {eq }} / \mathrm{cm}^{2}$, Nucl. Instr. Meth. A 612 (2010) 474.

[20] J. Lange, J. Becker, E. Fretwurst, R. Klanner, \& G. Lindstrom, Properties of a radiation-induced charge multiplication region in epitaxial silicon diodes, Nucl. Instr. Meth. A 622 (2010) 49.

[21] G. Casse, A. Affolder, P. Allport, H. Brown \& M. Wormald, Enhanced efficiency of segmented silicon detectors of different thicknesses after proton irradiation up to $1 \times 10^{16} n_{e q} \mathrm{~cm}^{-2}$, Nucl. Instr. Meth. A 624 (2010) 401.

[22] G. Kramberger, V. Cindro, I. Mandic, M. Mikuz, M. Milovanovic, M. Zavrtanik, \& K. Zagar, Investigation of Irradiated Silicon Detectors by Edge-TCT, IEEE Trans. Nucl. Sci. 57 (2010) 2294.

[23] G. Casse, A. Affolder, P. Allport \& M. Wormald, Evaluation of Floating Zone and Epitaxial Planar Silicon Detectors With Different Substrate Thickness After Irradiation up to $2 \times 10^{16} n_{\text {eq }} \mathrm{cm}^{-2}$, IEEE Trans. Nucl. Sci. 56 (2009) 3752.

[24] T. Latham, et. al., Performance of the LHCb Vertex Locator, to be published in the proceeding of TIPP2011, Chicago, IL.

[25] A. Seiden, Layout Options, ATLAS Tracker Upgrade Workshop, Liverpool, 6-8 Dec 2006, http://www.liv.ac.uk/physics/AHLUTW/

[26] N. Unno, et. al., Development of n-on-p silicon sensors for very high radiation environments, Nucl. Instr. Meth. A 636 (2011) S24.

[27] J. Bohm, et. al., Evaluation of the bulk and strip characteristics of large area $n$-in-p silcion sensors intended for a very high radiation environment, Nucl. Instr. Meth. A 636 (2011) S104.

[28] M. Ravnik \& R. Jeraj, Research reactor benchmarks, Nucl. Sci. Eng. 145 (2003) 145.

[29] K. Deiters, piE1 beam line, http://aea.web.psi.ch/beam2lines/beam_pie1.html

[30] A. Dierlamm, Studies on the Radiation Hardness of Silicon Sensors, IEKP-KA/03-23, Universitat Karlsruhe (TH), 2003.

[31] Y. Unno, et. al., p-bulk silicon microstrip sensors and irradiation, Nucl. Instr. Meth. A 579 (2007) 614. 
[32] K. Hara, et. al., Testing of bulk radiation damage of n-in-p silicon sensors for very high radiation environments, Nucl. Instr. Meth. A 636 (2011) S83.

[33] S. Lindgren, et. al., Testing of surface properties pre-rad and post-rad of n-in-p silicon sensors for very high radiation environment, Nucl. Instr. Meth. A 636 (2011) S111.

[34] H. F.-W. Sadrozinski, et. al., Punch-through protection of SSDs in beam accidents, Nucl. Instr. Meth. A, doi:10.1016/j.nima.2011.06.085.

[35] I. Mandic, et. al., Annealing effects in n+-p strip detectors irradiated with high neutron fluences, Nucl. Instr. Meth. A 629 (2011) 101.

[36] K.-H. Hoffmann, Campaign to identify the future CMS tracker baseline, Nucl. Instr. Meth. A., doi:10.1016/j.nima.2011.05.028.

[37] M. Glaser, L. Durieu, F. Lemeilleur, M. Tavlet, C. Leroy, \& P. Roy, New irradiation zones at the CERN PS, Nucl. Instr. Meth. A 426 (1999) 72.

[38] A. Macchiolo, et. al., Performance of Silicon n-in-p Pixel Detectors irradiated up to $5 \times 10^{15} n_{e q} \mathrm{~cm}^{-2}$ for future ATLAS Upgrades, to be published in the proceeding of TIPP2011, Chicago, IL.

[39] D. Forshaw, et. al., Characterization of Micron pixel detectors with analogue readout after neutron irradiation, presented at the 18th RD50 Workshop, Liverpool, May 23-25, 2011, https://indico.cern.ch/conferenceDisplay.py?confId=129737.

[40] A. Macchiolo, et. al., Performance of thin pixel sensors irradiated up to a fluence of $1 \times 10^{16} \mathrm{n}_{\mathrm{eq}} \mathrm{cm}^{-2}$ and development of a new interconnection technology for the upgrade of the ATLAS pixel system, Nucl. Instr. Meth. A 650 (2011) 145.

[41] Y. Unno, et. al., Development of n-in-p silicon planar pixel sensors and fip-chip modules for very high radiation environments, Nucl. Instr. Meth. A 650 (2011) 129.

[42] G. Dingemans, et. al., Comparison between aluminum oxide surface passivation films deposited with thermal ALD, plasma ALD and PECVD, 35th IEEE Photovoltaic Specialists Conference, doi:10.1109/PVSC.2010.5614508.

[43] M. Christophersen, et. al., Laser-Scribing and Sidewall Passivation of P-Type Sensors, 6th Trento Workshop, 2-4 March 2011, FBK, Povo di Trento, Italy.

[44] M. Povoli, et. al., Development of planar detectors with active edge, Nucl. Instr. Meth. A, doi:10.1016/j.nima.2011.04.050.

[45] X. Wu, Recent Results of VTT's Edgeless Detector Prototypes, to be published in the proceeding of iWoRID 2011, July 3-7th, 2011, Zurich, Switzerland.

[46] P. Fernandex-Martinez, et. al., Simulation of new p-type strip detectors with trench to enhance the charge multiplication effect in the n-type electrodes, Nucl. Instr. Meth. A, doi:10.1016/j.nima.2011.04.056. 